\title{
The SCD - Stem Cell Differentiation ESA Project: Preparatory Work for the Spaceflight Mission
}

\author{
Silvia Versari ${ }^{1}$ - Livia Barenghi ${ }^{1,2} \cdot{\text { Jack van } \operatorname{Loon}^{3} \cdot \text { Silvia Bradamante }}^{1}$
}

Received: 19 May 2015 / Accepted: 19 October 2015 / Published online: 12 November 2015

(C) The Author(s) 2015. This article is published with open access at Springerlink.com

\begin{abstract}
Due to spaceflight, astronauts experience serious, weightlessness-induced bone loss because of an unbalanced process of bone remodeling that involves bone marrow mesenchymal stem cells (BMSCs), as well as osteoblasts, osteocytes, and osteoclasts. The effects of microgravity on osteocells have been extensively studied, but it is only recently that consideration has been given to the role of BMSCs. Previous researches indicated that human BMSCs cultured in simulated microgravity $(\mathrm{sim}-\mu \mathrm{g})$ alter their proliferation and differentiation. The spaceflight opportunities for biomedical experiments are rare and suffer from a number of operative constraints that could bias the validity of the experiment itself, but remain a unique opportunity to confirm and explain the effects due to microgravity, that are only partially activated/detectable in simulated conditions. For this reason, we carefully prepared the SCD - STEM CELLS DIFFERENTIATION experiment, selected by the European Space Agency (ESA) and now on the International Space Station (ISS). Here we present the preparatory studies performed on ground to adapt the project to the spaceflight
\end{abstract}

\footnotetext{
Silvia Bradamante

silvia.bradamante@gmail.com

1 CNR-ISTM, Institute of Molecular Science and Technologies, Milan, Italy

2 Integrated Orthodontic Services s.r.l., Lecco, Italy

3 DESC (Dutch Experiment Support Center), Department Oral and Maxillofacial Surgery / Oral Pathology, VU-University Medical Center \& Academic Centre for Dentistry Amsterdam (ACTA), Amsterdam, The Netherlands
}

constraints in terms of culture conditions, fixation and storage of human BMSCs in space aiming at satisfying the biological requirements mandatory to retrieve suitable samples for post-flight analyses. We expect to understand better the molecular mechanisms governing human BMSC growth and differentiation hoping to outline new countermeasures against astronaut bone loss.

Keywords Microgravity · International Space Station · Epigenetics · Transcriptomics · Bone · Osteoporosis . Aging

$\begin{array}{ll}\text { Abbreviations } \\ \text { AT-MSCs } & \begin{array}{l}\text { Adipose Tissue derived Mesenchymal Stem } \\ \text { Cells }\end{array} \\ \text { BMSCs } & \begin{array}{l}\text { Bone Marrow Mesenchymal Stem Cells } \\ \text { CC }\end{array} \\ \text { Culture Chamber } \\ \text { EU } & \text { Experimental Unit } \\ \text { ESA } & \text { European Space Agency } \\ \text { EH } & \text { Experiment Hardware } \\ \text { FM } & \text { Flight Model } \\ \text { GM } & \text { Ground Model } \\ \text { ISS } & \text { International Space Station } \\ \text { MSCs } & \text { Mesenchymal Stem Cells } \\ \mu \mathrm{g} & \text { Microgravity } \\ \text { OM } & \text { Osteogenic Medium } \\ \text { RPM } & \text { Random Positioning Machine } \\ \text { sim- } \mu \mathrm{g} & \text { Simulated microgravity } \\ \text { SCs } & \text { Stem Cells } \\ \text { SM } & \text { Standard Medium } \\ \text { Vit D3 } & \text { 1,25-dihydroxy vitamin D3 }\end{array}$




\section{Introduction}

The goal of sending people farther in space, extending the duration of missions from months to years, requires space medicine to face new challenges. Longterm exposure to microgravity $(\mu \mathrm{g})$ produces in astronauts a number of physio-pathological alterations leading to problems such as motion sickness, cardiovascular deconditioning, muscle atrophy, and bone demineralization. Many of these diseases parallel the aging pathologies, but they occur and develop much more rapidly in space (Vernikos and Schneider 2009). One of the most critical diseases suffered by astronauts is bone loss. The studies of the bone loss observed in astronauts after spaceflight (Orwoll et al. 2013) and in ground simulated microgravity ( $\mathrm{sim}-\mu \mathrm{g}$ ) experiments (bed rest, hindlimb unloading, as well as in vitro studies of cellular models) have been extensively reviewed by Nagaraja and Risin (Nagaraja and Risin 2013). The phenomenon begins immediately on arrival in space. It has been observed that bone density decrease is about $1-2 \%$ per month in weight bearing bones, truly accelerated compared to the 2-3\% loss per year observed in postmenopausal females.

Most physiological effects due to short duration spaceflights resolve shortly after return to earth, but bone demineralization can be a permanent and dangerous consequence of long-duration spaceflight. During the past decade, researchers considered and investigated failures in osteoblast and osteoclast activity as responsible for bone mass loss in $\mu \mathrm{g}$ (Blaber et al. 2013). Now, the focus is on Stem Cells (SCs), which play a major role in the maintenance of bone mass, being the main source of osteoblasts during bone remodeling and repair (Senarath-Yapa et al. 2014; Rosset et al. 2014). The recruitment of an adequate number of osteoblasts is dependent on the availability of Mesenchymal Stem Cells (MSCs) and their proper response to growth, differentiation, and chemotactic signals in the microenvironment. Recent papers investigated the behavior of MSCs in sim- $\mu \mathrm{g}$ using ground simulators such as the Rotating Wall Vessel and the Random Positioning Machine (RPM). Although some discrepancies in results exist due to differences in origin of MSCs, bioreactors and differentiation stimuli, it is clear that sim- $\mu \mathrm{g}$ profoundly affects MSC behavior in terms of proliferation, differentiation, and senescence (Bradamante et al. 2014). While many cell types have already been tested in orbital flight, cultured human MSCs have never experienced real $\mu \mathrm{g}$. In 2009, based on the bone researches performed at that time (van Loon et al. 1996; Hughes-Fulford 2004; Knippenberg et al. 2005; Hughes-Fulford et al. 2006; Knippenberg et al. 2006; Versari et al. 2007; Bacabac et al. 2007, 2008; Knippenberg et al. 2009), we presented a spaceflight proposal to the "International Life Science Research Announcement 2009" (ILSRA 2009) convinced that a spaceflight experiment is the only way to clarify the influence of real $\mu \mathrm{g}$ on human MSC behavior and to help the identification of the molecular mechanisms for reversing bone loss that may be translated to human osteoporosis and in general to aged related bone pathologies. There is increasing evidence that most of the diseases related to aging are associated with a progressive decline in the number and/or function of SCs (Fukada et al. 2014; Oh et al. 2014). The goal of our original proposal was a general one, being focused also on tissue engineering applications; nevertheless, our spaceflight experiment had to be restricted to the study of the alterations in microgravity of human MSCs derived from bone marrow due to some limitations in the offered spaceflight opportunity. Our project named SCD - STEM CELL DIFFERENTIATION (previously ARIES) was selected by the European Space Agency (ESA) for the Soyuz 42S spaceflight mission schedule on March 27 2015. The experiment has been already in space and is currently analyzed. Here we report the results of the exhaustive ground study performed to prepare, in terms of biological and engineering requirements, our experiment aimed at investigating the effects of spaceflight on human BMSCs. In details, the ground study was focused mainly on: a) optimization of cell culture conditions to maintain cell viability in the proposed experiment hardware $(\mathrm{EH})$; b) feasibility of the proposed spaceflight protocol and optimization of the osteogenic medium; c) efficiency of the non-toxic fixative NOTOXhisto in samples preservation and in fixing cells for Oil Red $\mathrm{O}$ and Alizarin Red staining procedures.

Recent researches have shown the beneficial effects of hypoxia on proliferation/differentiation of MSCs (Estrada et al. 2012; Hung et al. 2012) and different lines have been combined toward the identification of major space genes (Clement 2012) During the preparation period, in line with the above mentioned papers we investigated also the influence of oxygen concentration in the cultivation of human MSCs in sim- $\mu \mathrm{g}$ (Versari et al. 2013a) using the genomic approach.

\section{Materials and Methods}

Human bone marrow MSCs (BMSCs) were purchased from Lonza, Allendale, NJ, USA. Cells were positive for CD105, CD166, CD29, and CD44 and negative for CD14, CD34 and CD45.

Cells were cultured on Thermanox collagen coated coverslips for 14 days either in Standard Medium (SM) or in Osteogenic Medium (OM) the composition of which 
Fig. 1 SCD - Stem Cell

Differentiation mission profile

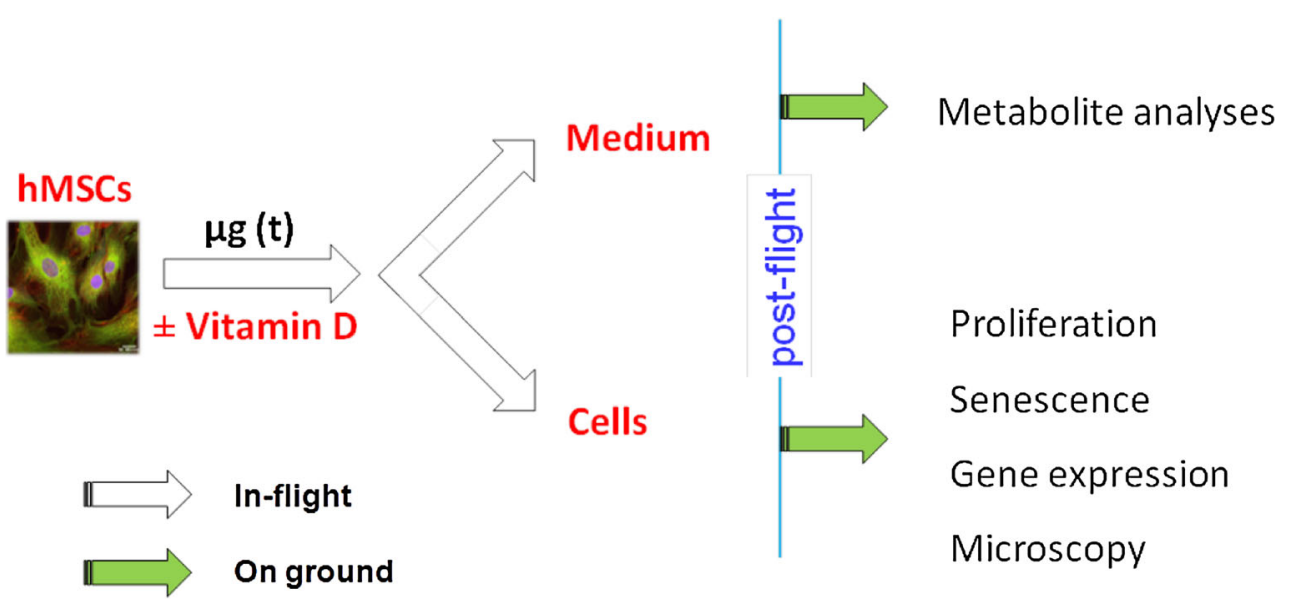

were the following: a) SM: DMEM (Dulbecco's Modified Eagle's medium) supplemented with $12.5 \mathrm{mM}$ HEPES, $10 \mathrm{U} / \mathrm{mL}$ heparin, $200 \mathrm{mM}$ glutamine, $500 \mu \mathrm{g} / \mathrm{mL}$ streptomycin sulphate, $600 \mu \mathrm{g} / \mathrm{mL}$ penicillin; b) OM: SM added with $0.1 \mathrm{M}$ ascorbic acid, $10 \mathrm{mM}$ b-glycerophosphate, and $10^{-8}$ M 1,25 dihydroxy vitaminD3 (Vit D3). All chemicals were purchased from Sigma Aldrich, Saint Louis, MO, USA. Thermanox coverslips were purchased from Thermo Fisher Scientific Inc. Waltham, MA USA. Due to safety constraint in space, we selected NOTOXhisto (Scientific Device Laboratory, USA) as non-toxic fixative.

To evaluate the influence of oxygen in culturing human BMSCs we repeated the experiments already performed on human Adipose tissue derived MSCs (AT-MSCs) (Versari et al. 2013a). Briefly, to simulate microgravity, we used the RPM, a device in which the gravity vector is continually reoriented with direction and speed randomization (maximum velocity of $60 \%$ s). In our conditions, the maximum 'residual $\mathrm{g}$ ' was expected to be $10^{-4} \mathrm{~g}$
(Borst and van Loon 2009). Cells were seeded in OptiCells ${ }^{\mathrm{TM}}$ fully filled with the medium previously degassed and then enriched with the selected oxygen concentration (5\% or $21 \%$ ). Then, Opticells ${ }^{\mathrm{TM}}$ were accommodated inside sealed metallic vessels filled either with a $5 \%$ $\mathrm{CO}_{2}$ /air mixture $\left(\mathrm{O}_{2}\right.$ concentration in the range 20-21\%) or $5 \% \mathrm{CO}_{2}: 5 \% \mathrm{O}_{2}: 90 \% \mathrm{~N}_{2}$ mixture and subjected to sim$\mu \mathrm{g}$ or accommodated on the bottom platform of the RPM as static controls ( $1 \mathrm{~g})$. At day 7 , medium was quickly changed stopping the RPM for no longer than $5 \mathrm{~min}$. Four experimental conditions were considered: 1) $1 \mathrm{~g}$ at $5 \% \mathrm{O}_{2}$, 2) $1 \mathrm{~g}$ at $\left.20 \% \mathrm{O}_{2}, 3\right)$ sim- $\mu \mathrm{g}$ at $\left.5 \% \mathrm{O}_{2}, 4\right)$ sim- $\mu \mathrm{g}$ at $20 \% \mathrm{O}_{2}$.

A focused array, the Human Mesenchymal Stem Cell $\mathrm{RT}^{2}$ Profiler ${ }^{\mathrm{TM}}$ PCR Array (SABiosciences) that profiles the expression of 84 key genes involved in human MSC pluripotency, self-renewal status and differentiation has been used to evaluate the effects of the different experimental conditions. The analysis of statistically significant gene expression changes was performed with the SABiosciences
Scheme 1 SCD spaceflight experiment scenario

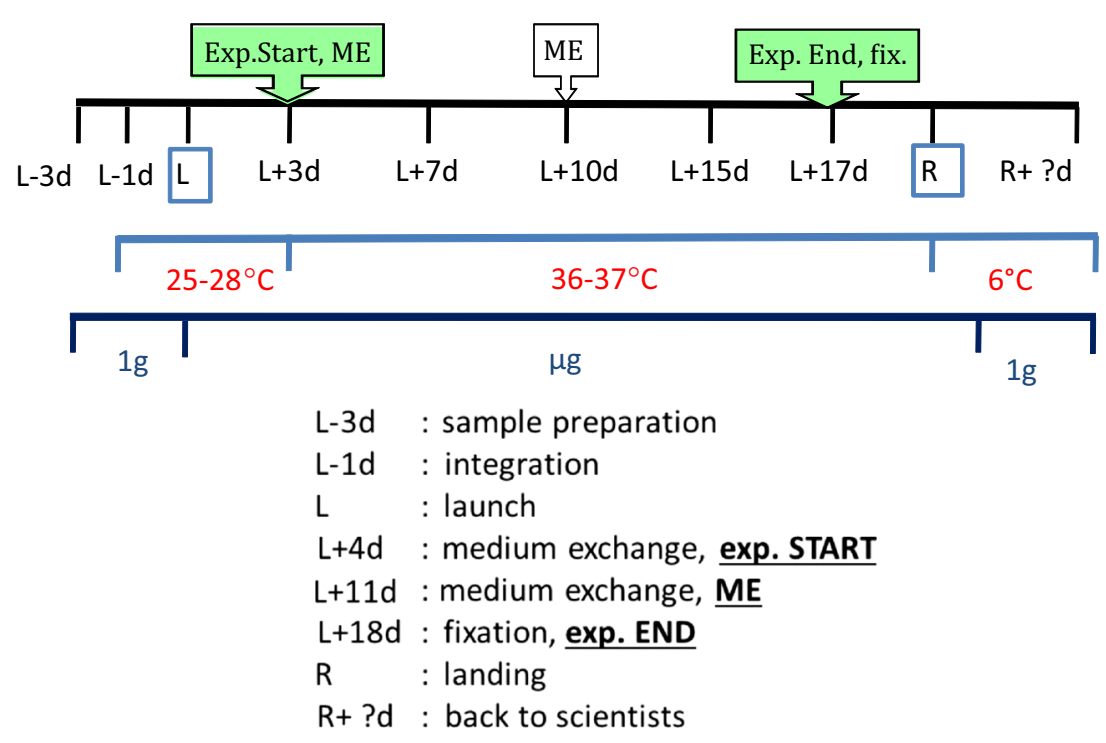


PCR Array Data analysis tool. This web-based software automatically performs all $\Delta \Delta \mathrm{Ct}$ based fold-change calculations from the uploaded raw threshold cycle (CT) data. The threshold cycle indicates the fractional cycle number at which the mount of amplified target reaches a fixed threshold. A 2-fold change cut-off was selected to identify the genes hose expression was significantly differentially regulated.

\section{Stem Cell Differentiation Flight Experiment Global Profile}

During the SCD experiment, human BMSCs will be incubated in $\mu \mathrm{g}$ for several days at conditioned temperature with regular medium refresh. Standard and osteogenic medium will be used. At the end of the active phase of the experiment, cells and medium will be separated, suitably fixed and stored for post-flight analyses as shown in the diagram of Fig. 1.

Human BMSCs will be cultured in the SCD Experimental Unit (EU) developed by Kayser Italia (Livorno, Italy; http://kayser.it/), that were already used in the ESASPHINX experiment (Versari et al. 2013b). The SCD EU consists of a brick made of biologically compatible plastic [polyetheretherketone (PEEK)] containing 5 cylinders (fluid-reservoirs, $1.8 \mathrm{ml}$ each), a culture chamber (CC, $230 \mathrm{~mm}^{2}$ ), and connecting channels. Five small valves were placed to separate the different fluids and the CC. Each cylinder has a piston (not shown) that, when released by a preloaded spring, injects the fluid into the $\mathrm{CC}$; the waste medium is collected in the previously emptied cylinder and suitably preserved. During the experiment (Scheme 1), all the medium exchanges and fixation operations were automated on the basis of a predefined timeline.

\section{Results and Discussion}

We here report the definition and optimization of some of the SCD experimental procedures and approaches in order

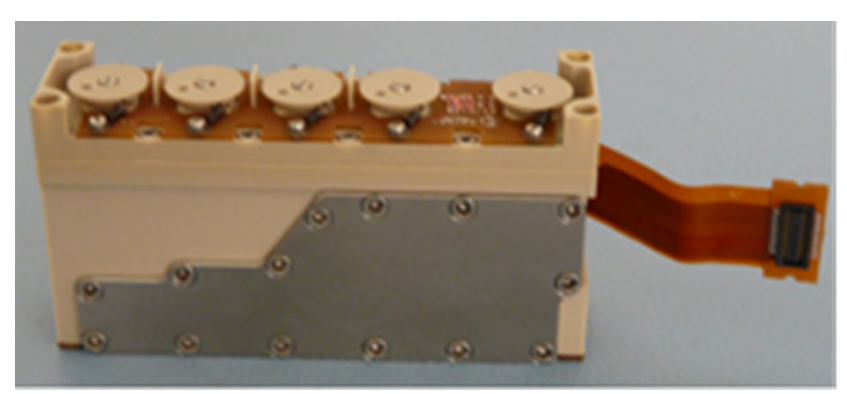

Fig. 2 SCD Experimental Unit (EU) with integrated electronics to successfully achieve the objective of the SCD study i.e. to determine how human BMSCs react to a prolonged (approx. 2 weeks) exposure to microgravity in terms of growth, senescence and differentiation toward osteoblasts when treated with Vit D3. A SCD spaceflight experiment possible scenario is reported in Scheme 1. During the experiment, all the medium exchanges and fixation operations will be automated based on a predefined timeline. 4 days before the launch (L) ground control (GM, n = 12) and spaceflight $(\mathrm{FM} \mathrm{n}=12)$ modules will be prepared on the launch site to be ready for handover. After the 4 days estimated to be necessary to reach the ISS, to be installed in KUBIK and to be warmed again to $37^{\circ} \mathrm{C}$, the SCD experiment will start $\left(T_{0}\right)$ with a first medium exchange (ME). The second ME will be after 7 days $\left(T_{0}+7\right.$ days $)$ and third one with PBS only after 14 days $\left(T_{0}+14\right.$ days). The experiment will be stopped by means of 2 subsequent RNAlater fixations (Sigma-Aldrich) separated by an interval of 6 min. After each exchange, the exhausted culture medium will be fixed using a protease inhibitor cocktail (Sigma-Aldrich). After the experiment will be completed, the $\mathrm{EH}$ modules will be kept at $6{ }^{\circ} \mathrm{C}$ inside the KUBIK incubator or moved in MELFI (Minus Eighty-Degree Laboratory Freezer for ISS) operating at $+2{ }^{\circ} \mathrm{C}$, before being returned to Earth. The 12 identical $\mathrm{EH}$ control modules prepared at the launch site will be run in parallel using the same experimental protocol. At the end, each module will provide a $230-\mathrm{mm}^{2}$ cell sample and the related preserved media.

\section{Optimization of Cell Culture Conditions to Maintain Cell Viability in the Proposed Experimental Hardware (EH)}

Being the proposed hardware (Fig. 2) totally sealed, it is mandatory to buffer the medium by adding HEPES to the culture medium. We tested whether the addition of HEPES to the culture medium could affect cell viability. In details, human BMSCs (low passage number) were seeded on Thermanox coverslips in SM added with HEPES, loaded in the SCD bioreactors and cultured at $37{ }^{\circ} \mathrm{C}$ for 2 days. Two different HEPES concentrations were tested: $12.5 \mathrm{mM}$ and $25 \mathrm{mM}$. At the end of the experiment, cells were trypsinized, stained with Trypan blue solution $(0.4 \%)$ and the viable cells were counted using a Burker chamber. The results in cell viability were compared with those obtained in standard culture conditions $\left(5 \% \mathrm{CO}_{2}\right.$, no HEPES). As shown in Fig. 3a, no significant difference in terms of cell number was found between HEPES-added medium and standard conditions. The $12.5 \mathrm{mM}$ HEPES was selected as optimal concentration for the spaceflight experiment as $25 \mathrm{mM}$ HEPES seems to affect, although slightly, cell viability. 

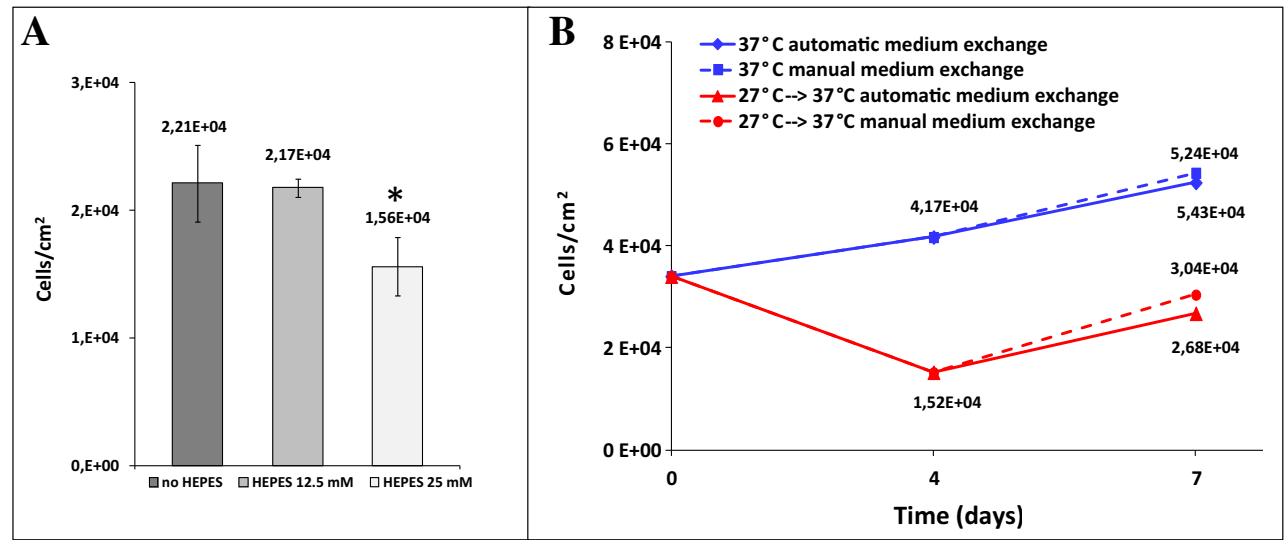

Fig. 3 A) Cell viability in HEPES-added SM for 2 days at $37^{\circ} \mathrm{C}$. $\mathrm{N}=12$; *no HEPES vs. HEPES $25 \mathrm{mM} \mathrm{p}<0.05$. B) Cell proliferation simulating the selected spaceflight protocol: 4 days at $27{ }^{\circ} \mathrm{C}$

\section{Testing the Feasibility of the Proposed Spaceflight Protocol}

Human BMSCs (low passage number) were seeded on Thermanox coverslips, loaded in the SCD EU and cultured simulating the proposed spaceflight protocol: 4 days at $27{ }^{\circ} \mathrm{C}$ (Soyuz scenario utilized until 2014, from launch to installation in KUBIK, today reduced to 2 days) followed by 3 days at $37{ }^{\circ} \mathrm{C}$. At day 4, medium was changed automatically (using the release system) or manually (with a pipette). Cell number was determined at day 4 and 7.

Four days culturing in the bioreactors at $27{ }^{\circ} \mathrm{C}$ profoundly affects cell viability (Fig. 3b). Nevertheless, when the bioreactors are moved to $37^{\circ} \mathrm{C}$, cell number is sufficient to resume proliferation, thus guaranteeing the feasibility of the spaceflight protocol and enough material for post-flight analyses. In addition, no significant difference between

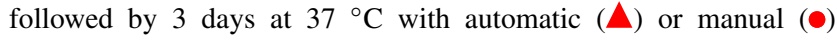
medium exchange at day 4 . Control experiments: 7 days at $37^{\circ} \mathrm{C}$ with automatic $(\$)$ or manual ( $\square$ ) medium exchange at day 4

automatic or manual medium exchange was found, thus confirming that the pressure exerted during the automatic fluid exchange was not detaching cells.

\section{Optimization of the Osteogenic Medium}

The standard protocol for MSC osteogenic differentiation requires OM to be refreshed every 3 days (Aslan et al. 2006; Boland et al. 2004). SCD spaceflight hardware and protocol request $\mathrm{OM}$ to be refreshed every 7 days, thus affecting osteogenic differentiation efficiency (Fig. 4, middle). We tested whether doubling osteogenic stimuli concentration in the OM overcomes this experimental constrain. In details, confluent human BMSCs (low passage number) were seeded on 6-well plates and incubated for 14 days with the two different osteogenic stimuli concentrations (ascorbic acid phosphate, $\beta$-glycerophosphate, and Vit D3): $1 \mathrm{X}$ and $2 \mathrm{X}$. OM was refreshed at day 7 . At the end

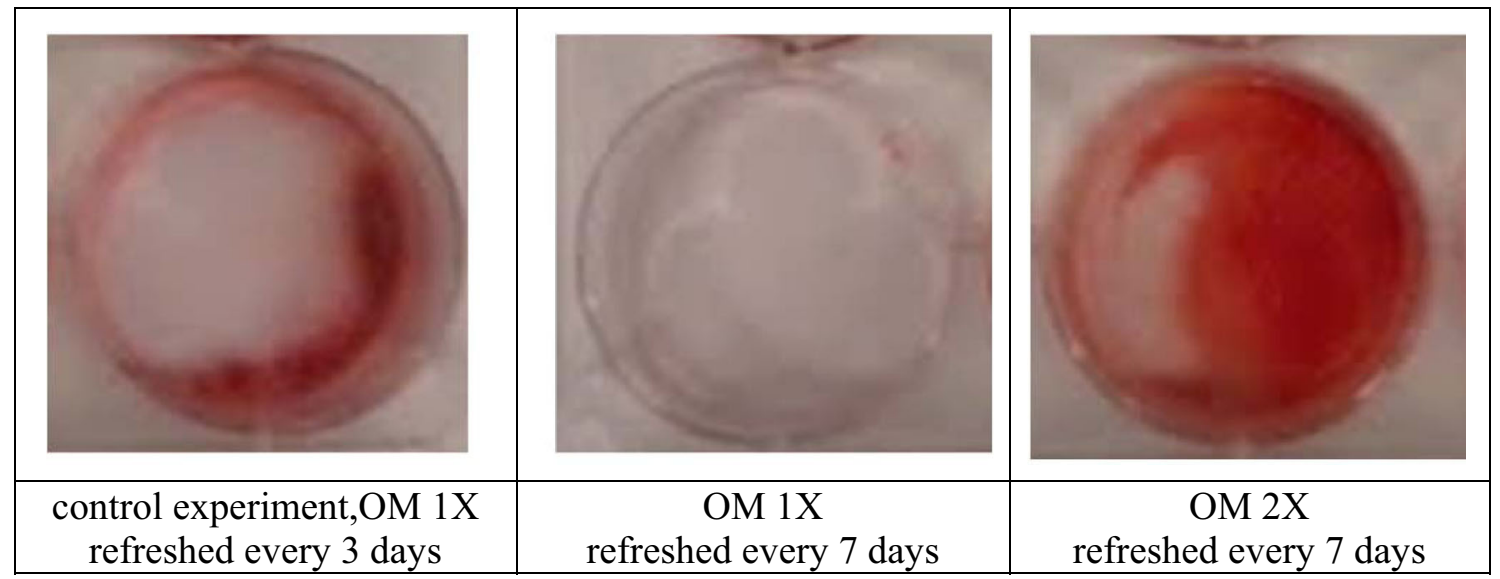

Fig. 4 Alizarin Red staining of human BMSCs at day 14. (Left): control experiment, OM 1X refreshed every 3 days; (Middle) OM 1X and (Right) OM $2 X$ refreshed every 7 days. OM $2 X$ changed every 7 days is capable of inducing osteogenic cell differentiation 
of the experiment, osteogenic differentiation was evaluated with Alizarin Red staining and results were compared with the control experiment (OM 1X, medium refreshed every 3 days).

As shown in Fig. 4, OM 2X (right) refreshed every 7 days is as efficient as the control OM $1 \mathrm{X}$ refreshed every 3 days (left) in the induction of osteogenic differentiation, thus overcoming the experimental constrain.

\section{Efficiency of the Non-toxic Fixative NOTOXhisto: A) in Samples Preservation Towards PFA One Month After Fixation; B) in Fixing Cells for Oil Red O and Alizarin Red Staining Procedures}

Standard fixation protocol in current laboratory practice requires the use of paraformaldehyde (PFA, Sigma). PFA is a very toxic fixative and, to be used in a spaceflight experiment, requires additional levels of containment and,

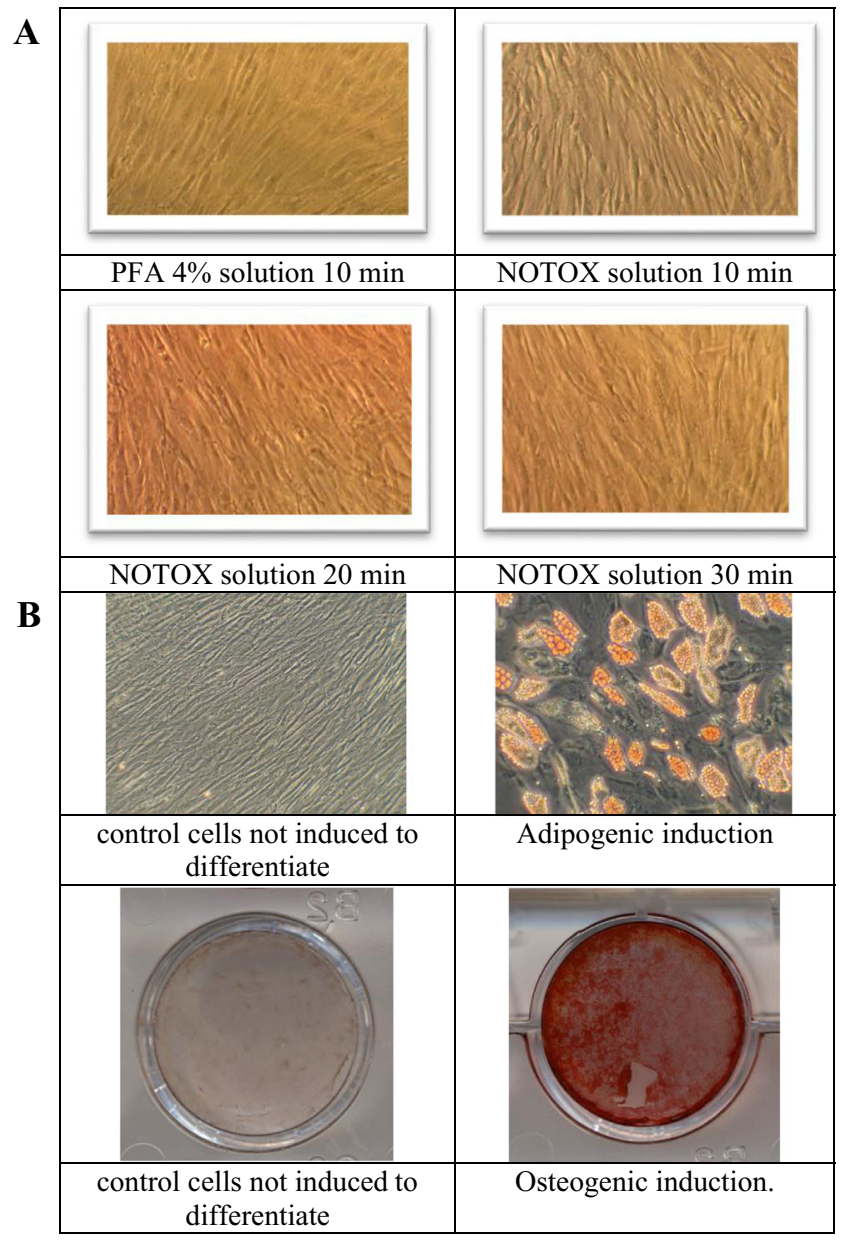

Fig. 5 A Images of human BMSCs one month after fixation with: PFA $4 \%$ for $10 \mathrm{~min}$; and NOTOXhisto for $10 \mathrm{~min}$; $20 \mathrm{~min}$; and $30 \mathrm{~min}$. Microscope magnification: 10X; camera magnification: 4X. B Images of human BMSCs after Oil Red O and Alizarin Red staining consequently, additional costs for EH improvement. For this reason, we tested whether the non-toxic fixative NOTOXhisto was suitable for the preservation of the sample in our spaceflight experiment. In details, human BMSCs were seeded in 6-well plates at a density of $20.000 \mathrm{cells} / \mathrm{cm}^{2}$ and cultured in SM. At confluence, cells were washed with PBS and fixed with $4 \%$ PFA solution in PBS for $10 \mathrm{~min}$ or NOTOXhisto solution (Scientific Device Laboratory, USA) for 10, 20 and $30 \mathrm{~min}$. After fixation, cells were washed and stored in PBS at $4{ }^{\circ} \mathrm{C}$ for 1 month, and examined with a conventional inverted microscope. As shown in Fig. 5a, after one month storage cells were in very good shape and no differences were found between cells fixed in PFA $4 \%$ and cells fixed in NOTOXhisto. The same cells, at confluence, cultured as before in SM, were treated for 14 days with adipogenic or osteogenic medium to induce cell differentiation. At the end of the experiment, cells were washed with PBS, fixed with NOTOXhisto solution for $10 \mathrm{~min}$ and analysed with conventional microscopy after Oil Red O or Alizarin Red staining, markers of adipogenic or osteogenic differentiation, to evaluate the presence of lipid droplets or calcium deposition respectively. As shown in Fig. 5b, the staining procedure after fixation with NOTOXhisto was working properly.

\section{Effects of Oxygen Concentration: the Genomic Approach}

In vivo, human MSCs reside in specific "perivascular niches" (Moore and Lemischka 2006) and the various tissues where these cells are found are characterized by a low oxygen tension $\left(\mathrm{pO}_{2}\right)$ of about 2-8\% (Kofoed et al. 1985; Harrison et al. 2002; Matsumoto et al. 2005; Pasarica et al. 2009; Mohyeldin et al. 2010). It has been shown that $\mathrm{pO}_{2}$ plays a key role in regulating $\mathrm{SC}$ fate (Csete 2005; Panyukhin et al. 2008). Since the effects of

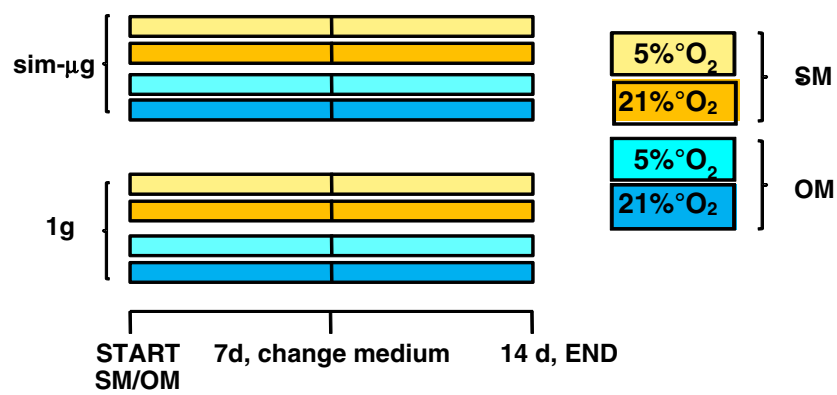

Scheme 2 Experimental protocol applied to compare the proliferation and differentiation responses of human BMSCs cultured in different gravity and oxygen concentration conditions. $1 \mathrm{~g}=$ ground control; sim- $\mu \mathrm{g}=$ microgravity simulated with the RPM; SM = standard medium; $\mathrm{OM}=$ osteogenic medium ( $\mathrm{SM}+$ ascorbic acid phosphate, $\beta$-glycerophosphate, and Vit D3); $\mathrm{d}=$ days 

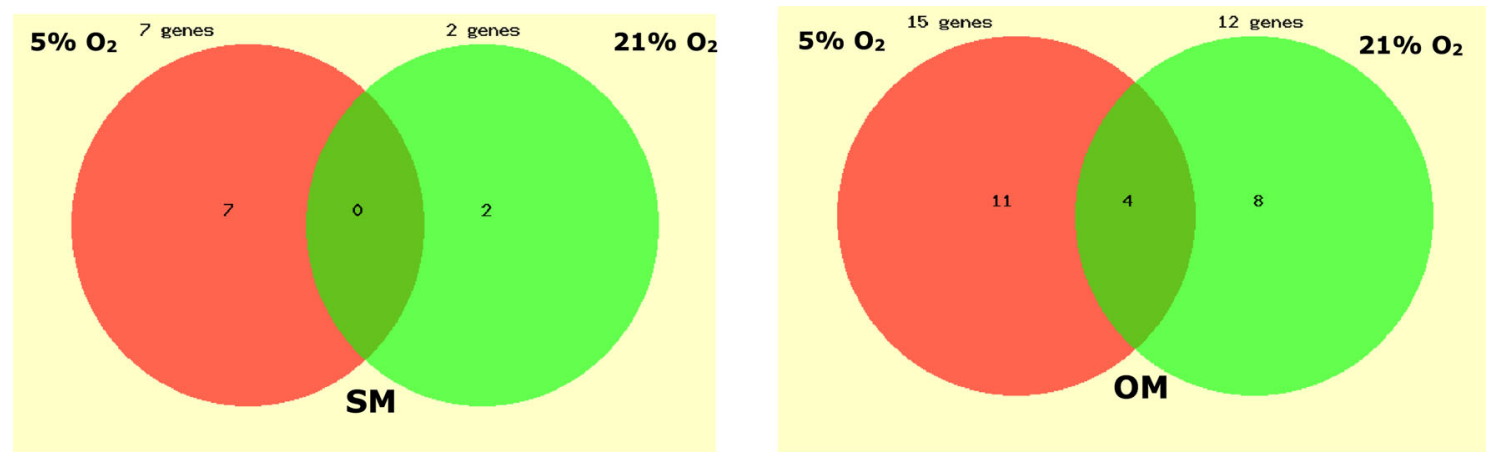

Fig. 6 VENN diagram showing the number of genes enriched in sim- $\mu \mathrm{g}$ vs. $1 \mathrm{~g}$ at $5 \% \mathrm{O}_{2}$ (red circle) and $21 \% \mathrm{O}_{2}$ (green circle) in the $\mathrm{SM}$ (left panel) and OM (right panel) and their overlaps. A 2-fold change cut-off was set to identify genes whose expression was significantly differentially regulated

"reduced $\mathrm{pO}_{2}$ " in the cultivation of human MSCs in sim- $\mu \mathrm{g}$ was not considered in the past, we performed a wide and interesting analysis on human AT-MSCs cultured at different oxygen concentration and g levels (Versari et al. 2013a) to investigate whether different $\mathrm{O}_{2}$ tensions modulate their gene expression profiles. We concluded that oxygen seems to play a marked role in amplifying the alterations in "adhesion and communication" pathways, common features in all of the sim- $\mu \mathrm{g}$ experiments. Considering the actual interest in the role of oxygen concentration in the proliferation, differentiation, and senescence events (Boyette et al. 2014), we evaluated whether different $\mathrm{O}_{2}$ tensions in sim- $\mu \mathrm{g}$ affect also human BMSCs that we selected for the SCD experiment. We chose a focused array, the Human Mesenchymal Stem Cell RT ${ }^{2}$ Profiler ${ }^{\mathrm{TM}}$ PCR Array (SABiosciences) that profiles the expression of 84 key genes involved in human MSC pluripotency, self-renewal status and differentiation. In details, human BMSCs (low passage number) were subjected to the two previously tested oxygen concentrations $\left(5 \% \mathrm{O}_{2}\right.$ and $\left.21 \% \mathrm{O}_{2}\right)$ in $\mathrm{SM}$ or $\mathrm{OM}$ in sim- $\mu \mathrm{g}$ using the
RPM (Scheme 2) for 14 days. The results were compared with those obtained at $1 \mathrm{~g}$ in the same conditions (ground controls).

At day 14 samples were harvested and a) Proliferation was measured with trypan blue cell counting; b) Cell death was evaluated with ToxiLight ${ }^{\mathrm{TM}}$ BioAssay kit; c) Total RNA was extracted, purified, and Human Mesenchymal Stem Cell $\mathrm{RT}^{2}$ Profiler ${ }^{\mathrm{TM}}$ was performed. Oxygen concentration in sim- $\mu \mathrm{g}$ influences human BMSC gene expression profile, but does not affect proliferation and cell death (data not shown). As shown in Fig. 6 in SM a) at $5 \%$ $\mathrm{O}_{2} 7$ genes were found significantly modulated in sim$\mu \mathrm{g}$ compared to the related $1 \mathrm{~g}$ controls, whereas b) at $21 \% \mathrm{O}_{2} 2$ genes. No overlap between the two comparisons was found (left panel). In $\mathrm{OM}$ a) at $5 \% \mathrm{O}_{2}$ 15 genes were found significantly modulated in sim- $\mu \mathrm{g}$ compared to the related $1 \mathrm{~g}$ controls, whereas b) at $21 \%$ $\mathrm{O}_{2} 12$ genes. An overlap of 4 genes, that represent the genes enriched in both oxygen conditions, was found (right panel).
Table 1 List of the genes significantly modulated in sim- $\mu \mathrm{g}$ vs.1g in the two comparisons analysed $\left(5 \% \mathrm{O}_{2}\right.$ and $21 \% \mathrm{O}_{2}$ ) in $\mathrm{SM}$

\begin{tabular}{lllll}
\hline Gene name & $5 \% \mathrm{O}_{2} \mathrm{SM}$ & $\begin{array}{l}21 \% \mathrm{O}_{2} \mathrm{SM} \\
\mathrm{FC}\end{array}$ & Function & Annotation \\
\hline COL1A1 & n.m. & $-2,3$ & hMSC specific & Collagen, type I, alpha 1 \\
CD44 & 2,2 & n.m. & hMSC specific & CD44 molecule \\
HGF & 2,1 & n.m. & hMSC specific & Hepatocyte growth factor \\
KITLG & 2,1 & n.m. & hMSC specific & KIT ligand \\
NT5E & 2,0 & n.m. & hMSC specific & CD73 \\
MCAM & $-2,5$ & n.m. & hMSC specific & Melanoma cell adhesion molecule \\
JAG1 & n.m. & $-2,3$ & Miogenesis & Jagged 1 \\
GDF5 & 2,4 & n.m. & Tenogenesis & Growth differentiation factor 5 \\
RUNX2 & 2,0 & n.m. & Osteogenesis & Runt-related transcription factor 2 \\
\hline
\end{tabular}

$F C$ fold change, n.m. not modulated 
Table 2 List of the genes significantly modulated in sim- $\mu \mathrm{g}$ vs. $1 \mathrm{~g}$ in the two comparisons analysed $\left(5 \% \mathrm{O}_{2}\right.$ and $21 \% \mathrm{O}_{2}$ ) in $\mathrm{OM}$

\begin{tabular}{|c|c|c|c|c|}
\hline Gene name & $\begin{array}{l}5 \% \mathrm{O}_{2} \mathrm{OM} \\
\mathrm{FC}\end{array}$ & $\begin{array}{l}21 \% \mathrm{O}_{2} \mathrm{OM} \\
\mathrm{FC}\end{array}$ & Function & Annotation \\
\hline FGF2 & 3,0 & 3,7 & Stemness & Fibroblast growth factor 2 \\
\hline LIF & n.m. & 4,1 & Stemness & Leukemia inhibitory factor \\
\hline BDNF & n.m. & 2,2 & hMSC specific & Brain-derived neurotrophic factor \\
\hline ENG & n.m. & 18,7 & hMSC specific & Endoglin \\
\hline IL6 & n.m. & 2,4 & hMSC specific & Interleukin 6 (interferon, beta 2) \\
\hline NUDT6 & 4,8 & 3,1 & hMSC specific & Nudix-type motif 6 \\
\hline NES & n.m. & $-2,6$ & hMSC specific & Nestin \\
\hline PDGFRB & n.m. & $-2,7$ & hMSC specific & Platelet-derived growth factor receptor $\beta$ \\
\hline TGFB3 & 2,2 & $-4,0$ & hMSC specific & Transforming growth factor, $\beta 3$ \\
\hline THY1 & - & $-2,5$ & hMSC specific & Thy- 1 cell surface antigen \\
\hline VEGFA & 3,6 & $-18,9$ & hMSC specific & Vascular endothelial cell Growth factor $\alpha$ \\
\hline ALCAM & 5,1 & n.m. & hMSC specific & Activated leukocyte cell adhesion mol \\
\hline CASP3 & 2,9 & n.m. & hMSC specific & Caspase 3 \\
\hline CTNNB1 & 2,5 & n.m. & hMSC specific & Catenin (cadherin-associated protein) $\beta 1$ \\
\hline ITGAV & 2,7 & n.m. & hMSC specific & Integrin, alpha $\mathrm{V}$, antigen CD51 \\
\hline ITGB1 & 3,6 & n.m. & hMSC specific & Integrin, beta 1 , antigen $\mathrm{CD} 29$ \\
\hline MITF & 2,2 & n.m. & hMSC specific & Microphthalmia-ass transcription factor \\
\hline NT5E & 3,6 & n.m. & hMSC specific & 5'-nucleotidase, ecto (CD73) \\
\hline SMURF2 & 4,0 & n.m. & Osteogenesis & SMAD specific E3 ubiquitin prot ligase 2 \\
\hline SMAD4 & 2,4 & n.m. & Tenogenesis & SMAD family member 4 \\
\hline SOX9 & 2,0 & n.m. & Chondrogenesis & SRY (sex determining region Y)-box 9 \\
\hline HAT1 & 4,2 & n.m. & Chondrogenesis & Histone acetyltransferase 1 \\
\hline GDF15 & n.m. & 3,3 & Chondrogenesis & Growth differentiation factor 15 \\
\hline
\end{tabular}

$F C$ fold change, n.m. not modulated
The most significant changes were found in some specific pluripotency and self-renewal genes (e.g. CD44, CD73, HGF, COL1A1, FGF2, LIF, IL6, TGFB3, VEGFA) and differentiation (GDF5, GDF15, RUNX2, SMURF2, SMAD4, SOX9, HAT1) markers. See Tables 1 and 2 for details.

These preliminary results point to oxygen as an important factor influencing human MSC behaviour in sim- $\mu \mathrm{g}$. It is noteworthy that differences in oxygen concentration induce the modulation of genes not only involved in hypoxia/hyperoxia response, but also in self-renewal, pluripotency, and differentiation.

In Table 2, in hyperoxia is worth noting A) a marked up-regulation of endoglin (CD105), a gene coding for a Type I homodimeric transmembrane glycoprotein which binds with high affinity transforming growth factor (TGF)- 31 and TGF- $\$ 3$. In addition there is evidence that endoglin plays an important role in the dedifferentiation mechanism (Barbara et al. 1999; Frobel et al. 2014). It is noteworthy that the overexpression of endoglin markedly reduces osteogenic differentiation of MSC as detected by Alizarin Red staining (private communication),B) a marked down-regulation of Vascular Endothelial Growth Factor (VEGF). This is an interesting result since VEGF is an essential coordinator of extracellular matrix remodeling, angiogenesis and bone formation (Schipani et al. 2009).

\section{Conclusion}

The SCD - Stem Cell Differentiation experiment addresses an important question: how human MSCs respond to the real microgravity environment. Based on these preliminary tests and on the positive outcome of the optimization of the spaceflight experimental procedures and analytical techniques, we are confident that the aims of the application will be achieved. We here anticipate that the specific benefits of the conceived space experiment will range from a better understanding of the molecular mechanisms governing human BMSC growth and differentiation to the possibility of outlining new countermeasures against astronaut bone 
loss. The potential fall-out on ground of the STEM CELL DIFFERENTIATION experiment concerns both the fields of age-related bone pathologies and tissue engineering.

Acknowledgments This work was supported by the European Space Agency (contract number $4200014651 / \mathrm{SH} / \mathrm{NH} / \mathrm{NL} /==\mathrm{CCN} 004$ ) and Kayser Italia S.r.l.

Open Access This article is distributed under the terms of the Creative Commons Attribution License which permits any use, distribution, and reproduction in any medium, provided the original author(s) and the source are credited.

\section{References}

Aslan, H., Zilberman, Y., Kandel, L., Liebergall, M., Oskouian, R.J., Gazit, D., Gazit, Z.: Osteogenic differentiation of noncultured immunoisolated bone marrow-derived CD105 cells. Stem Cells 24, 1728-1737 (2006)

Bacabac, R.G., Mizuno, D., Schmidt, C.F., MacKintosh, F.C., Van Loon, J.J.W.A., Klein-Nulend, J., Smit, T.H.: Round versus flat: bone cell morphology, elasticity, and mechanosensing. J. Biomech. 41, 1590-1598 (2008)

Bacabac, R.G., Van Loon, J.J.W.A., KleinNulend, J.: Microgravity and bone cell mechanosensitivity. Biology in Space and Life on Earth: Effects of Spaceflight on Biological Systems. pp. 157-177 (2007)

Barbara, N.P., Wrana, J.L., Letarte, M.: Endoglin is an accessory protein that interacts with the signaling receptor complex of multiple members of the transforming growth factor $\beta$ superfamily. J. Biol. Chem. 274, 584-594 (1999)

Blaber, E.A., Dvorochkin, N., Lee, C., Alwood, J.S., Yousuf, R., Pianetta, P., Globus, R.K., Burns, B.P., Almeida, E.A.: Microgravity induces pelvic bone loss through osteoclastic activity, osteocytic osteolysis, and osteoblastic cell cycle inhibition by CDKN1a/p21. PLoS One 8, e61372 (2013). doi: 10.1371/journal. pone.0061372

Boland, G.M., Perkins, G., Hall, D.J., Tuan, R.S.: Wnt 3a promotes proliferation and suppresses osteogenic differentiation of adult human mesenchymal stem cells. J. Cell. Biochem. 93, 1210-1230 (2004)

Borst, A.G., van Loon, J.J.W.A.: Technology and developments for the random positioning machine, RPM. Microgravity Sci. Technol. 21, 287-292 (2009)

Boyette, L.B., Creasey, O.A., Guzik, L., Lozito, T., Tuan, R.S.: Human bone marrow-derived mesenchymal stem cells display enhanced clonogenicity but impaired differentiation with hypoxic preconditioning. Stem Cells Translational Medicine 3, 241 (2014)

Bradamante, S., Barenghi, L., Maier, J.A.M.: Stem cells toward the future: the space challenge. Life 4, 267-280 (2014)

Clement, J.Q.: Gene expression microarrays in microgravity research: toward the identification of major space genes. INTECH Open Access Publisher (2012)

Csete, M.: Oxygen in the cultivation of stem cells. Ann. N. Y. Acad. Sci. 1049, 1-8 (2005). doi:10.1196/annals.1334.001

Estrada, J.C., Albo, C., Benguria, A., Dopazo, A., LopezRomero, P., Carrera-Quintanar, L., Roche, E., Clemente, E.P., Enriquez, J.A., Bernad, A.: Culture of human mesenchymal stem cells at low oxygen tension improves growth and genetic stability by activating glycolysis. Cell Death Differ. 19, 743-755 (2012)

Frobel, J., Hemeda, H., Lenz, M., Abagnale, G., Joussen, S., Denecke, B., $\AA$ arï̈, T., Zenke, M., Wagner, W.: Epigenetic rejuvenation of mesenchymal stromal cells derived from induced pluripotent stem cells. Stem Cell Rep. 3, 414-422 (2014)

Fukada, S.-i., Ma, Y., Uezumi, A.: Adult stem cell and mesenchymal progenitor theories of aging. Frontiers in Cell and Developmental Biology 2, 1-9 (2014)

Harrison, J.S., Rameshwar, P., Chang, V., Bandari, P.: Oxygen saturation in the bone marrow of healthy volunteers. Blood 99, 394 (2002)

Hughes-Fulford, M.: Signal transduction and mechanical stress. Science Signaling 2004. re12 (2004)

Hughes-Fulford, M., Rodenacker, K., Jtting, U.: Reduction of anabolic signals and alteration of osteoblast nuclear morphology in microgravity. J. Cell. Biochem. 99, 435-449 (2006)

Hung, S.P., Ho, J.H., Shih, Y.R.V., Lo, T., Lee, O.K.: Hypoxia promotes proliferation and osteogenic differentiation potentials of human mesenchymal stem cells. J. Orthop. Res. 30, 260-266 (2012)

Knippenberg, M., Helder, M.N., Doulabi, B.Z., Bank, R.A., Wuisman, P.I.J.M., Klein-Nulend, J.: Differential effects of bone morphogenetic protein- 2 and transforming growth factor- $\hat{I}^{2} 1$ on gene expression of collagen-modifying enzymes in human adipose tissue-"derived mesenchymal stem cells. Tissue Eng. A 15, 22132225 (2009)

Knippenberg, M., Helder, M.N., Doulabi, B.Z., Wuisman, P., KleinNulend, J.: Osteogenesis versus chondrogenesis by BMP-2 and BMP-7 in adipose stem cells. Biochem. Biophys. Res. Commun. 342, 902-908 (2006)

Knippenberg, M., Helder, M.N., Zandieh Doulabi, B., Semeins, C.M., Wuisman, P.I.J.M., Klein-Nulend, J.: Adipose tissue-derived mesenchymal stem cells acquire bone cell-like responsiveness to fluid shear stress on osteogenic stimulation. Tissue Eng. 11, 1780-1788 (2005)

Kofoed, H., Sjontoft, E., Siemssen, S.O., Olesen, H.P.: Bone marrow circulation after osteotomy. Blood flow, pO2, pCO2, and pressure studied in dogs. Acta Orthop. Scand. 56, 400-403 (1985)

Matsumoto, A., Matsumoto, S., Sowers, A.L., Koscielniak, J.W., Trigg, N.J., Kuppusamy, P., Mitchell, J.B., Subramanian, S., Krishna, M.C., Matsumoto, K.: Absolute oxygen tension (pO(2)) in murine fatty and muscle tissue as determined by EPR. Magn. Reson. Med. 54, 1530-1535 (2005). doi:10.1002/mrm.20714

Mohyeldin, A., Garzon-Muvdi, T., Quinones-Hinojosa, A.: Oxygen in stem cell biology: A critical component of the stem cell niche. Cell Stem Cell 7, 150-161 (2010). doi:10.1016/j.stem.2010.07.007

Moore, K.A., Lemischka, I.R.: Stem cells and their niches. Science 311, 1880-1885 (2006). doi:10.1126/science. 1110542

Nagaraja, M.P., Risin, D.: The current state of bone loss research: data from spaceflight and microgravity simulators. J. Cell. Biochem. 114, 1001-1008 (2013). doi:10.1002/jcb.24454

Oh, J., Lee, Y.D., Wagers, A.J.: Stem cell aging: mechanisms, regulators and therapeutic opportunities. Nat. Med. 20, 870-880 (2014)

Orwoll, E.S., Adler, R.A., Amin, S., Binkley, N., Lewiecki, E.M., Petak, S.M., Shapses, S.A., Sinaki, M., Watts, N.B., Sibonga, J.D.: Skeletal health in long-duration astronauts: nature, assessment, and management recommendations from the NASA Bone Summit. J. Bone Miner. Res. 28, 1243-1255 (2013). doi:10.1002/jbmr.1948

Panyukhin, N.V., Vishnyakova, K.S., Yegorov, Y.E.: Effect of partial oxygen pressure on survival, proliferation, and differentiation of mouse bone marrow mesenchymal stem cells. Biochemistry (Moscow) Supplemental Series A: Membrane and Cell Biology 2, 326-332 (2008)

Pasarica, M., Sereda, O.R., Redman, L.M., Albarado, D.C., Hymel, D.T., Roan, L.E., Rood, J.C., Burk, D.H., Smith, S.R.: Reduced 
adipose tissue oxygenation in human obesity: evidence for rarefaction, macrophage chemotaxis, and inflammation without an angiogenic response. Diabetes 58, 718-725 (2009). doi: $10.2337 / \mathrm{db} 08-1098$

Rosset, P., Deschaseaux, F., Layrolle, P.: Cell therapy for bone repair. Orthop. Traumatol. Surg. Res. 100, S107-S112 (2014)

Schipani, E., Maes, C., Carmeliet, G., Semenza, G.L.: Regulation of osteogenesisangiogenesis coupling by HIFs and VEGF. J. Bone Miner. Res. 24, 1347-1353 (2009)

Senarath-Yapa, K., McArdle, A., Renda, A., Longaker, M.T., Quarto, N.: Adipose-derived stem cells: a review of signaling networks governing cell fate and regenerative potential in the context of craniofacial and long bone skeletal repair. Int. J. Mol. Sci. 15, 9314-9330 (2014)

van Loon, J.J.W.A., Veldhuijzen, J.P., Burger, E.H.: Bone and space flight: an overview. In: Biological and medical research in space, pp. 259-299. Springer (1996)
Vernikos, J., Schneider, V.S.: Space, gravity and the physiology of aging: parallel or convergent disciplines? A mini-review. Gerontology 56, 157-166 (2009)

Versari, S., Klein-Nulend, J., van Loon, J., Bradamante, S.: Influence of oxygen in the cultivation of human mesenchymal stem cells in simulated microgravity: An explorative study. Microgravity Sci. Technol. 25, 59-66 (2013a)

Versari, S., Longinotti, G., Barenghi, L., Maier, J.A.M., Bradamante, S.: The challenging environment on board the International Space Station affects endothelial cell function by triggering oxidative stress through thioredoxin interacting protein overexpression: the ESA-SPHINX experiment. FASEB J. 27, 4466-4475 (2013b)

Versari, S., Villa, A., Helder, M.N., Doulabi, B.Z., van Loon, J., Bradamante, S.: Effects of gravity on proliferation and differentiation of adipose tissue-derived stem cells. Journal of gravitational physiology: a journal of the International Society for Gravitational Physiology 14, P127-128 (2007) 